\title{
Correspondence
}

Cerebrovascular Diseases

Cerebrovasc Dis 2011;32:513-514

DOI: $10.1159 / 000330701$

\section{Letter Referring to the Paper by Galinovic et al.: Fully Automated Postprocessing Carries a Risk of Substantial Overestimation of Perfusion Deficits in Acute Stroke Magnetic Resonance Imaging}

\author{
Yasmina Chaibi, Timothé Boutelier
}

Olea Medical, La Ciotat, France

We read the paper by Galinovic et al. [1] with great interest since it aims to address one of the major concerns in emergency stroke assessment regarding the reliability of the postprocessing software currently available in clinical practice and clinical research settings. Obviously, nowadays there is a certain amount of such postprocessing software pervading clinical practice, either via major equipment constructors or independent vendors, or as a result of research programs. However, for the medical doctor confronted with the daily practice and challenges associated with stroke assessment in the acute phase, the advantages and disadvantages of one software over another remain blurred since few developers of such tools provide transparent information on exactly which algorithms have been applied and what the main limitations might be, whether they be design dependent or algorithm dependent.

Therefore, although this study raised our interest since we are all users of such technology in our practice, we would like to express our concern about several biases showing in the methodology applied in this study. In the aforementioned study, the authors tried to demonstrate that fully automated postprocessing presents a high risk of overestimation of perfusion deficits in acute stroke patients, based on a sample of patients with no evidence of stroke. However, if - as explained by the authors - the main idea was to demonstrate that fully automated postprocessing erroneously indicated diffusion deficits where there were none, then the appropriate sample for the study should have consisted of healthy volunteers with radiologic evidence of no perfusion deficits instead of a widely heterogeneous group of patients with various cerebral diseases. Only such a sample of healthy subjects would have allowed them to test the original hypothesis of overestimation and eventually to quantify it as a limitation of deconvolution algorithms, which all tend to overestimate perfusion deficits due to the algorithm itself and thresholds [2-4].

Further, it has not become clear to us whether the authors wished to determine the impact of automation because they clearly stated in the paper that arterial input function was (1) manually selected by PerfScape, although the software offers a specifically designed function for automatic selection, and (2) automatically selected by PMA and Stroketool, then manually adjusted.
Also, regarding the automation of the calculation, skull and cerebrospinal fluid filtering is automatic in PerfScape, whereas it seems to be insufficient in the other software and thereby requires additional manipulation of calculated maps in the latter.

Additionally, the authors stated that different thresholds for mean transit time and cerebral blood flow were empirically chosen for each software, which again is inconsistent with an automated procedure. Also, the comparison of relative values for cerebral blood flow, without normalization, does not seem to be relevant. One could argue that the comparison of mean transit time and $\mathrm{T}_{\max }$ maps, supposedly absolute values, would be more appropriate. However, it is well known that $\mathrm{T}_{\max }$ is highly and directly dependent on the arterial input function selected, whereas the mean transit time is strongly dependent on the deconvolution method [4]. Therefore, a normalization step is mandatory prior to any attempt at comparison.

The original aim of the current study as stated by the authors was to highlight the overestimation of perfusion deficits due to fully automated postprocessing. In this regard, the statistical comparison between the three software applications by means of Spearman correlation tests between diffusion and perfusion volumes has no relevance. As explained above, without prior normalization, relative values cannot be compared. Besides, this statistical analysis can neither reject nor confirm the original hypothesis of the study.

In the light of these remarks, we agree with the authors on the potential of improvement of most of the available software. However, although these softwares are not perfect yet, the benefit provided by automatic postprocessing of perfusion parameters in clinical settings has already been demonstrated by several teams, showing promise especially for stroke assessment in emergency units [5-9].

\section{References}

1 Galinovic I, Brunecker P, Ostwaldt AC, Soemmer C, Hotter B, Fiebach JB: Fully automated postprocessing carries a risk of substantial overestimation of perfusion deficits in acute stroke magnetic resonance imaging. Cerebrovasc Dis 2011;31:408-413.

2 Ostergaard L, Weisskoff RM, Chesler DA, Gyldensted C, Rosen BR: High resolution measurement of cerebral blood flow using intravascular trace bolus passage. Part I. Mathematical approach and statistical analysis. Magn Reson Med 1996;36:715-725.

-3 Perthen JE, Calamante F, Gadian DG, Connelly A: Is quantification of bolus tracking MRI reliable without deconvolution? Magn Reson Med 2002;47:61-67.

-4 Kudo K, Sasaki M, Yamada K, Momoshima S, Utsunomiya H, Shirato $\mathrm{H}$, Ogasawara K: Differences in CT perfusion maps generated by different commercial software: quantitative analysis by using identical source data of acute stroke patients. Radiology 2010;254:200-209.

5 Campbell BC, Christensen S, Foster SJ, Desmond PM, Parsons MW, Butcher KS, Barber PA, Levi CR, Bladin CF, Donnan GA, Davis SM: Visual assessment of perfusion-diffusion mismatch is inadequate to select patients for thrombolysis. Cerebrovasc Dis 2010;29:592-596.

\section{KARGER}

Fax +41613061234 E-Mail karger@karger.ch www.karger.com (c) 2011 S. Karger AG, Basel

$1015-9770 / 11 / 0325-0513 \$ 38.00 / 0$ 
6 Steve TA, Asdaghi N, Jeerakathil T, Knash ME, Hameed B, Beaulieu C, Shuaib A, Emery D, Butcher KS: Validation of an automated perfusiondiffusion lesion assessment tool (abstract). International Stroke Conference and Nursing Symposium Poster Presentations. Stroke 2011; 42:e288.

7 Luby M, Boparai S, Lynch JK, Merino JG, Latour L, Song SS, Hsia A, Warach S: Does threshold based segmentation of MRI correlate with visual reading of ischemia and perfusion deficit in acute scans (poster)? International Stroke Conference and Nursing Symposium Poster Presentations. Stroke 2011;42:e280.

8 Nicoli F, Squarcioni C, Grimaud L, Barberet M, Girard N: Microbubble administration during prolonged $2 \mathrm{MHz}$ TCD improves recanalization and long-term functional outcome after IV thrombolysis for MCA M1 occlusion (abstract). Abstracts of the World Stroke Congress, Seoul, 2010. Int J Stroke 2010;5(suppl 2):99.
9 Nicoli F, Squarcioni C, Grimaud L, Souleihet V, Barberet M, Girard N: Results of intravenous thrombolysis using exclusive MR imaging selection: a series of 420 acute ischemic stroke patients (abstract). Abstracts of the World Stroke Congress, Seoul, 2010. Int J Stroke 2010;5(suppl 2):99.

Yasmina Chaibi, $\mathrm{PhD}$

Olea Medical, ZI Athelia IV

93, avenue des Sorbiers

FR-13600 La Ciotat (France)

Tel. +33 44271 2420, E-Mail yasmina.chaibi@olea-medical.com 